\title{
IVOM in Österreich 2013 - Eine Auswertung anhand realer Patientenzahlen
}

\author{
Gerhard Kieselbach · Anna Vavrovsky · Ronald Hochreiter
}

Eingegangen: 10. Januar 2016 / Angenommen: 16. Januar 2016 / Online publiziert: 7. März 2016

(C) Die Autor(en) 2016. Dieser Artikel ist auf Springerlink.com mit Open Access verfügbar.

\section{Zusammenfassung}

Hintergrund Bereits bei einer ersten Untersuchung des ÖAC (Vavrovsky und Kieselbach, Value Health 15(4):A255, 2012) im Jahre 2011 wurde festgestellt, dass in Österreich pro Patient mit AMD nur 4 IVOM in einem Jahr erfolgten. Daher wurden in dieser Studie alle Indikationen pro Auge evaluiert.

Material und Methode Anhand von anonymisierten Akten von Patienten, die intravitreale Injektionen (IVOM) mit aVEGF Medikamenten wegen einer AMD, DMÖ oder RVV während des Jahres 2013 erhielten, wurde die Anzahl der IVOM pro Auge evaluiert. Zusätzlich wurde bei einem Teil der Visusverlauf innerhalb dieses Jahres untersucht.

Resultate Im Jahr 2013 wurden 54.321 IVOM durchgeführt, davon konnten 31.768 evaluiert werden. $67 \%$ aller IVOM in 29 Abteilungen erfolgten bei AMD (Altersbedingter Makuladegeneration). $21 \%$ der Patienten hatten ein DMÖ (diabetisches Makulaödem) und $12 \%$ wurden wegen einem RVV (retinalem Venenverschluss) behandelt. Die durchschnittliche Anzahl aller IVOM pro Jahr und Auge war 3.3 über alle drei Indikationen. Von 394 Patienten wurde auch der Visusverlauf erhoben. Es zeichnete sich eine klare Korrelation zwischen der Anzahl

ao. Univ.-Prof. Dr. G. Kieselbach $(\bowtie)$

Universitätsklinik für Augenheilkunde und Optometrie,

Medizinische Universität Innsbruck,

Anichstraße 35,

6020 Innsbruck, Österreich

E-Mail: gerhard.kieselbach@i-med.ac.at

Dr. A. Vavrovsky

Academy for Value in Health,

Wien, Österreich

PD Dr. R. Hochreiter

Institut für Statistik und Mathematik, Wirtschaftsuniversität Wien, Wien, Österreich an IVOM und dem Verlauf des Sehvermögens ab, die in allen rezenten Studien beschrieben wird (Holz et al., $\mathrm{Br}$ J Ophthalmol, doi:10.1136/bjophthalmol-2014-305327, 2014; Ziemssen et al., Ophthalmologe 112(3):246-254, 2015; Cohen et al., Retina 33:474-481, 2013; Holz et al., J Clin Invest 124:1430-1438, 2014).

Schlussfolgerung Die derzeitige IVOM Versorgung in Österreich ist nicht ausreichend, um das Sehvermögen der betroffenen Patienten zu erhalten. Die Verbesserung der Organisation und budgetären Ausstattung sowie insbesonders die Ausweitung in den niedergelassenen Bereich ist notwendig, um Erblindungen zu vermeiden.

Schlüsselwörter IVOM · aVEGF · AMD · DMÖ · RVV

Real life data of intravitreal injections in Austria in 2013

\section{Summary}

Background This study reports the real-life use of intravitreal injections of anti-vascular endothelial growth factor (aVEGF) therapy for AMD, DME and RVO throughout 1 year in Austria.

Material and methods Medical records of patients receiving intravitreal injections (IVOM) of anti-vascular endothelial growth factor (VEGF) therapy for AMD, DME and RVO between 1 January and 31 December 2013 were evaluated. Primary objective was to evaluate the number of IVOM per eye and year for all of the three indications. Secondary outcomes included changes in visual acuity during 1 year of anti-VEGF therapy.

Results In 201354.321 IVOM were identified, out of this number $67 \%$ of 31.768 evaluated IVOM in 29 departments were performed for AMD. $21 \%$ of patients were treated for DME and $12 \%$ received treatment for RVO. The mean number of injections over all three indications was 3.3 for eye and year. Out of 488 patients 
who received anti-VEGF injection, 394 were included for visual acuity assessment for the treated eye during 1 year. There was a clear correlation between number of IVOM and increase of visual acuity, similar to most of recent published studies (Holz et al., Br J Ophthalmol, doi:10.1136/bjophthalmol-2014-305327, 2014; Ziemssen et al., Ophthalmologe 112(3):246-254, 2015; Cohen et al., Retina 33:474-481, 2013; Holz et al., J Clin Invest 124:1430-1438, 2014).

Conclusions Treatment intensity was not sufficient to maintain visual acuity by anti VEGF treatment. Reallife results for visual acuity and injection frequency were worse at that time than in other countries (Holz et al., Br J Ophthalmol, doi:10.1136/bjophthalmol-2014-305327, 2014). Regularly treatment not only in hospitals is required to preserve visual acuity.

Keywords Intravitreal injections · aVEGF · AMD · DME • RVO

\section{Einleitung}

Die intravitreale operative Medikamentenapplikation IVOM ist mit über 55.000 Injektionen pro Jahr (2014) der häufigste operative Eingriff in Österreich. Dennoch wird diese hochwirksame Therapie von Politik und Gesundheitsbehörden konsequent nach unten lizitiert.

Um eine Grundlage für notwendige Gespräche mit den Verantwortlichen in Politik und in den Gremien zu erhalten, wurde bereits 2011 ein Vergleich der IVOM-Utilisationsraten mit MoH-Daten für 2010 durch eine Befragung von 26 Zentren (n IVOM=30.124) durchgeführt [1].

Der Vergleich mit den CATT Ergebnissen ergab, dass nur $30 \%$ der AMD Patienten eine State-of-the-Art Behandlung erhielten [2,3]. Allerdings wurde nicht eruiert, wie viele Augen betroffen waren. Die aktuellen Studienergebnisse der letzten zwei Jahre [4-6] zeigen unzweifelhaft, daß für den Erhalt des Sehvermögens über einen Zeitraum von mehr als 3 Jahren die durchschnittliche IVOM Frequenz pro Jahr von 5 IVOM $[3-5,7,8]$ nicht unterschritten werden darf. Dies gilt vor allem für die AMD, aber auch das DMÖ, benötigt nach bisherigem Wissenstand zum Visuserhalt mehr als 4 IVOM pro Jahr $[12,13]$

Daraus leitete sich natürlich die Frage ab, wie hoch die Gesamtanzahl der IVOM mit a-VEGF, unabhängig von der verwendeten Substanz, für die Gesamtanzahl von Augen, jeweils für die Indikationen AMD, DMÖ und RVO ist.

Um einen repräsentativen Querschnitt zu erhalten wurde ein Zeitfenster von einem Jahr, das rezente Jahr zwischen 1. Jänner und 31. Dezember 2013 gewählt.

\section{Methodik}

In einer Einzelpatientenanalyse an 12 Zentren in 8 Bundesländern wurden $31.768 \mathrm{im}$ Jahr 2013 durchgeführte IVOM nach Grunderkrankung, Indikation und Injektionsfrequenz per Auge analysiert.

Die vollständig anonyme Evaluierung an allen Zentren erfolgte vor Ort anhand der vorhandenen Aktenlage in einem Zeitraum von 5 Monaten. Zwei Abteilungen waren in der Lage aufgrund der bereits vorhandenen Daten die Fragen zu evaluieren und auszuwerten. An zwei Zentren wurde auch der Visusverlauf innerhalb des Beobachtungszeitraums erhoben und mit Alter, Diagnose und Zahl der IVOM korreliert.

Insgesamt wurden an den teilnehmenden Zentren im Jahr 2013 31.768 IVOM durchgeführt, die von uns evaluiert wurden. Zahlen des österreichischen Bundesministeriums für Gesundheitbenennen 54.321 IVOMin29 österreichischen Zentren, womit mit dieser Evaluierung eine Abdeckung von $58 \%$ aller durchgeführten IVOM in der vorliegenden Untersuchung entsprochen wird.

Alle relevanten Daten wurden aus den verschiedenen Krankenhaussystemen und Patientenbögen maskiert und nicht identifizierbar erhoben. Auch die Zentren wurden maskiert.

Die statistischen Auswertungen wurden mit Hilfe der Statistik-Software R erstellt. Dabei wurden vor allem die nötigen Datentransformationen durchgeführt, um die entsprechenden deskriptiven Statistiken zu berechnen. Insbesondere wurde dabei auf die Aufbereitung der Daten hinsichtlich der unterschiedlich auszuwertenden Attribute Wert gelegt, im Speziellen bei der Kombination bzw. Separation bezogen auf die unterschiedlichen Behandlungen des linken und rechten Auges der Patientinnen.

\section{Resultate}

Die Untersuchung zeigt, dass die AMD auch im Jahr 2013 der Grund für den Großteil der durchgeführten IVOM und konsistent über alle Zentren, Indikationen und Patienten war. $67 \%$ der durchgeführten IVOM waren AMD und $21 \%$ waren DMÖ, der Rest von $12 \%$ waren Venenverschlüsse.

Daraus errechnete sich eine im europäischen Vergleich $[4,7]$ sehr geringe Anzahl von Behandlungen pro Jahr. Im Mittel führte jedes evaluierte Zentrum im Jahr 20132647 IVOM durch. Das Zentrum mit der geringsten Anzahl durchgeführter IVOM verzeichnete 2013351 IVOM, jenes mit der größten IVOM Anzahl 5791 (Abb. 1).

Über alle Zentren, Indikationen und Patienten wurden 2013 durchschnittlich 4.2 pro Patient und 3.3 IVOM pro Auge über 12 Monate verabreicht (Abb. 2).

Insgesamt $26 \%$ der behandelten Patienten erhielten 6 oder mehr als 6 IVOM pro Jahr. Von 488 Patienten, die mehr als eine oder mehrere IVOM erhielten, wurde bei 394 Patienten das Sehvermögen vom 1.1. bis 31.12 evaluiert. Der Zusammenhang mit Visusverlauf und Anzahl der IVOM war ebenfalls eindeutig. Die Anzahl der IVOM korreliert positiv mit dem Visusverlauf innerhalb eines Jahres. Mit abnehmender Zahl an Injektionen fällt das Sehvermögen. 
Abb. 1 Die erste Säule zeigt den Durchschnittswert, die weiteren Säulen die anonymen Zentren

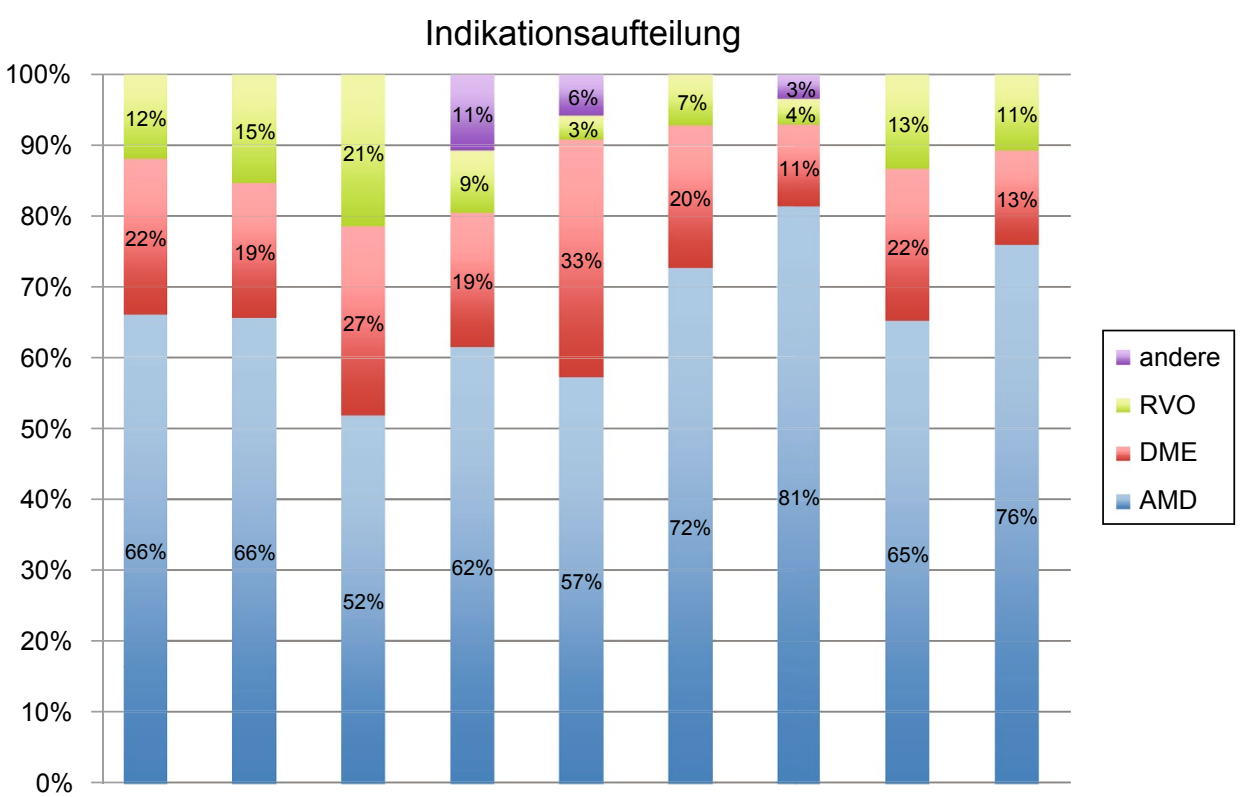

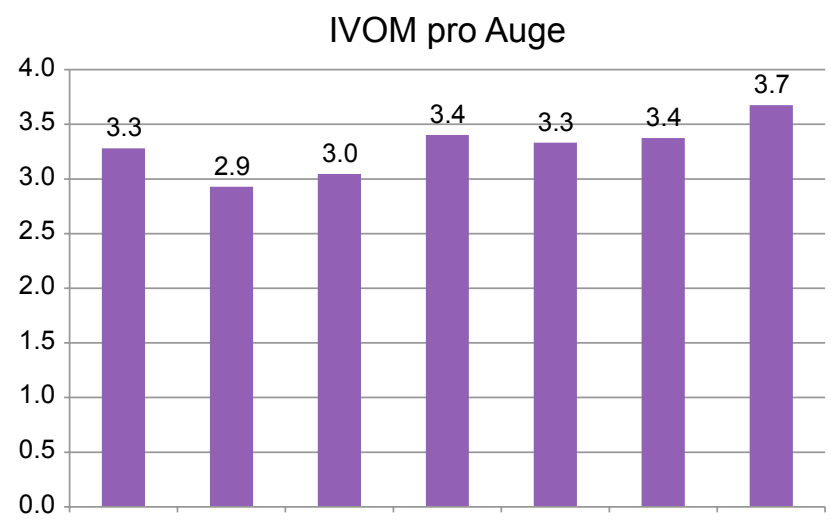

Abb. 2 Die erste Säule zeigt den Durchschnittswert, die weiteren Säulen die anonymen Zentren
Der Visusverlauf zeigt auch eine eindeutige Korrelation mit dem Alter, aber abhängig von der Anzahl der IVOM auf. Mit zunehmendem Alter sinkt die Anzahl der stabilen Augen, allerdings sind die Zahlen von Patienten über 80 sehr gering (Abb. 3).

\section{Diskussion}

Die gefundenen Visuswerte sind aufgrund der retrospektiven Evaluierung eines Behandlungsjahres statistisch nicht signifikant, decken sich aber mit den Auswertungen ähnlicher Studien, die in den letzten Jahren in den USA oder Europa publiziert wurden $[4,7$, 9-11]. Haupttenor aller Diskussionen ist die Tatsache, dass trotz der bekannten Studienzahlen die zum Visus-
Abb. 3 Visusänderung nach Anzahl IVOM pro Jahr und Auge

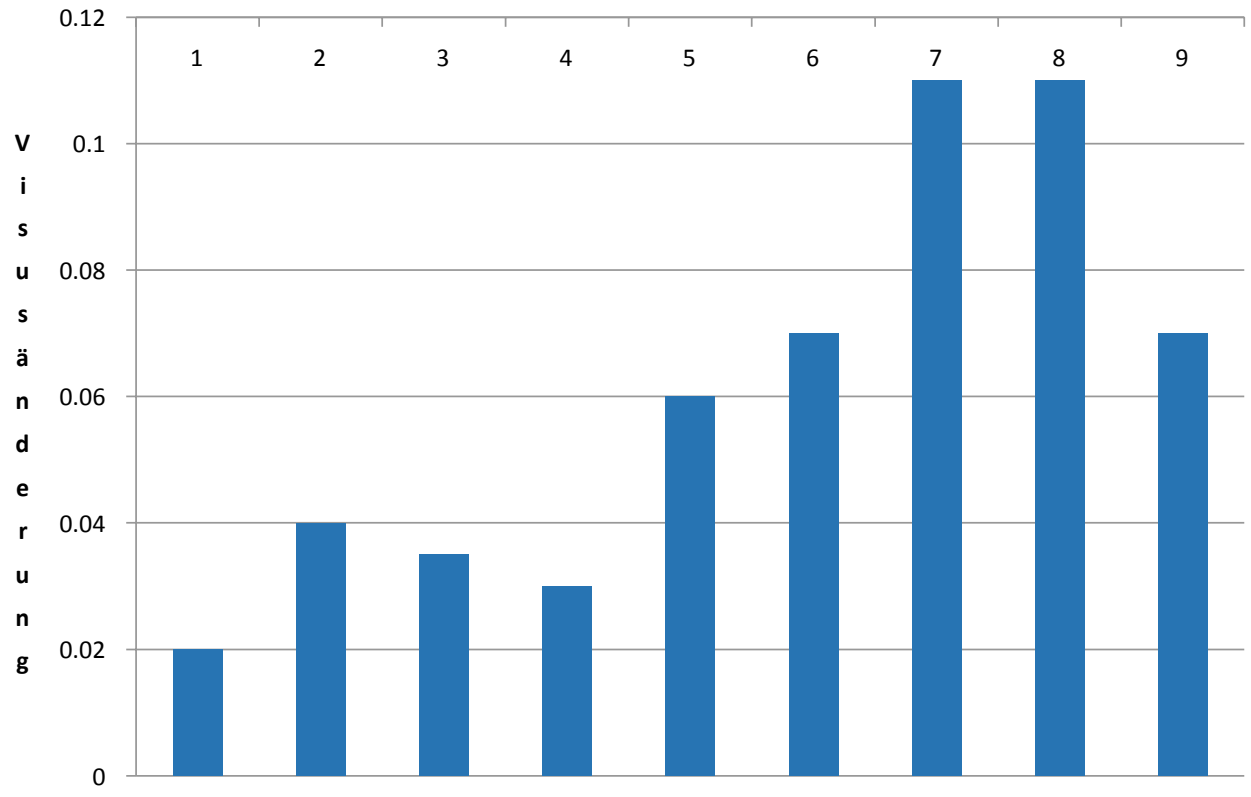


erhalt notwendigen IVOM nicht erreicht werden. Aus gesundheitsökonomischen Überlegungen ist besonders Interessant, dass das System in Irland (11 IVOM in 2a) und in den UK (9.0 in 2a) die besten Visusergebnisse bei der höchsten Zahl an IVOM erzielt. Ähnlich gute Zahlen weist noch Canada auf, während Deutschland mit 5.6 IVOM in 2a und Italien mit 5.2 IVOM in 2a noch schlechter sind als Österreich [4].

Der in Österreich gefundene Visusverlauf zeigt eine eindeutige Korrelation mit dem Alter, aber wieder abhängig von der Anzahl der IVOM auf. Genauso wie in der deutschen Studie „Behandlungsrealität von Ranibizumab bei nAMD in Deutschland" ein eindeutiger Zusammenhang zwischen Visusverlauf und Anzahl der Injektionen gezeigt wird [5].

Trotz der Daten aus den Zulassungsstudien, der österreichischen Manta Studie (1), der CATT Studie (2) und neuer Publikationen $[7,8]$ gelingt es in Österreich nur in maximal einem Drittel der Fälle ausreichend $\mathrm{zu}$ behandeln um das Sehvermögen $\mathrm{zu}$ verbessern oder zumindest zu erhalten. Mit zunehmendem Alter sinkt zudem die Anzahl der stabilen Augen, allerdings sind die Zahlen von Patienten über 80 mit mehr als 5 IVOM/a zu gering, um eine relevante Aussage geben zu können. Kein Unterschied im Visusverlauf ergab sich im Geschlechtervergleich. Ein weiterer limitierender Faktor ist sicher die retrospektive Auswertung von Visusdaten aus Ambulanzprotokollen und die fehlende Trennung von laufender Therapie und IVOM Beginnern.

Obwohl die finanziellen Voraussetzungen in Deutschland bis heuer deutlich besser als in Österreich waren, und die IVOM auch im extramuralem Bereich durchgeführt werden, sind die Zahlen sehr gering $[4,5]$.

Es bestand in der deutschen Studie auch ein Zusammenhang zwischen der Anzahl der IVOMs im ersten und zweiten Jahr und dem Therapieerfolg gemessen als absoluter Buchstabengewinn.

Insgesamt betrug in dieser Studie die Sehverbesserung (>0 Buchstabengewinn) im ersten Jahr $22 \%$ und im zweiten Jahr 13\%. Mit mehr als 7 IVOMs war im ersten Jahr eine Sehverbesserung bei $62,5 \%$ der Patienten eingetreten, mit 5 bis 7 IVOMs bei $44,7 \%$ und mit < 5 IVOMs bei $44,9 \%$.

Das bedeutet dass neben dem fehlenden finanziellen Anreiz auch ein organisatorischer Mangel besteht. US Studien belegen eindeutig, dass mit mehr Aufwand, personell und finanziell eine adäquate Versorgung möglich ist [9].

Das Bundesministerium für Gesundheit, der Hauptverband der Sozialversicherungen und die Privatversicherungen samt den zuständigen Landesräten für Gesundheit haben in den letzten Jahren die Patienten und Augenärzte ignoriert und die IVOM Jahr für Jahr herabgestuft, so daß heute diese Behandlung nicht mehr kostendeckend durchgeführt werden kann. Zugleich behauptete der Hauptverband noch im Mai dieses Jahres, daß die Versorgung der Bevölkerung ausreichend sei.

Im Sinne des Visus der Versorgten, aber auch der Kostenträger, die Präparate aufgrund der klinischen Wirksamkeit, die mittels der Zulassungsdosis erreicht wurde, erstatten, ist daher dringend eine Verbesserung der organisatorischen und budgetären Situation der Augenabteilungen, vor allem aber eine Ausweitung der Leistung IVOM in den extramuralen Raum zu fordern.

\section{Einhaltung ethischer Richtlinien}

\section{Interessenkonflikt}

G. Kieselbach gibt an, dass kein Interessenkonflikt besteht.

A. Vavrovsky gibt an, dass kein Interessenkonflikt besteht.

R. Hochreiter gibt an, dass kein Interessenkonflikt besteht.

\section{Open Access}

Dieser Artikel unterliegt den Bedingungen der Creative Commons Attribution License. Dadurch sind die Nutzung, Verteilung und Reproduktion erlaubt, sofern der/die Originalautor/en und die Quelle angegeben sind.

\section{Literatur}

1. Vavrovsky A, Kieselbach G. Intravitreal injections in Austria - results of an expert survey and comparison with utilisation rates from the Austrian ministry of health. Value Health. 2012;15(4):A255.

2. MANTA Research Group, Krebs I, Schmetterer L, Boltz A, Told R, Vécsei-Marlovits V, Egger S, et al. A randomised double-masked trial comparing the visual outcome after treatment with ranibizumab or bevacizumab in patients with neovascular AMD. Br J Ophthalmol. 2013;97(3):266-71.

3. CATT Research Group, Martin DF, Maguire MG, Ying GS, Grunwald JE, Fine SL, Jaffe GJ. Ranibizumab and bevacizumab for neovascular age-related macular degeneration. $\mathrm{N}$ Engl J Med. 2011;364(20):1897-908. www.med.upenn.edu/cpob/studies/documents / CATTEligibilityCriteria.pdf.

4. Holz FG, Tadayoni R, Beatty S, Berger A, Cereda MG, Cortez R, Hoyng CB, Hykin P, Staurenghi G, Heldner S, Bogumil T, Heah T, Sivaprasad S. Multi-country real-life experience of anti-vascular endothelial growth factor therapy for wet age-related macular degeneration. Br J Ophthalmol. 2014. doi:10.1136/bjophthalmol-2014-305327.

5. Ziemssen F, Eter N, Fauser S, Bopp S, Radermacher M, Hasanbasic Z, Holz FG, AURA-Studiengruppe. [Retrospective investigation of anti-VEGF treatment reality and effectiveness in patients with neovascular age-related macular degeneration (AMD) in Germany: treatment reality of ranibizumab for neovascular AMD in Germany]. Ophthalmologe. 2015;112(3):246-54. doi:10.1007/ s00347-014-3217-7.

6. Cohen SY, Mimoun G, Oubraham H, et al. Changes in visual acuity in patients with wet age-related macular degeneration treated with intravitreal ranibizumab in daily clinical practice: the LUMIERE study. Retina. 2013;33:474-81.

7. Holz FG, Schmitz-Valckenberg S, Fleckenstein M. Recent developments in the treatment of age-related macular degeneration. J Clin Invest. 2014;124:1430-8. 
8. Martin DF, Maguire MG, Fine SL, et al. Ranibizumab and bevacizumab for treatment of neovascular age-related macular degeneration: two-year results. Ophthalmology. 2012;119:1388-98.

9. Prennera JL, Halperinb LS, Rycroftc C, Hogued S, Williams Liu Z, Seiberte R. Disease burden in the treatment of agerelated macular degeneration: findings from a time-andmotion study. Am J Ophthalmol. 2015;160(4):725-31.el.

10. Keenan TD, Kelly SP, Sallam A, et al. Incidence and baseline clinical characteristics of treated neovascular age-related macular degeneration in a well-defined region of the UK. Br J Ophthalmol. 2013;97:1168-72.
11. Muether PS, Hoerster R, Hermann MM, et al. Long-term effects of ranibizumab treatment delay in neovascular age-related macular degeneration. Graefes Arch Clin Exp Ophthalmol. 2013;251:453-8.

12. Augood CA, Vingerling JR, De Jong PT, et al. Prevalence of age-related maculopathy in older Europeans: the European Eye Study (EUREYE). Arch Ophthalmol. 2006;124:529-35.

13. Chakravarthy U, Harding SP, Rogers CA, et al. Alternative treatments to inhibit VEGF in age-related choroidal neovascularisation: 2 -year findings of the IVAN randomised controlled trial. Lancet. 2013;382:1258-67. 